\title{
Consulta de Enfermagem às pessoas surdas: uma análise contextual
}

\author{
Nursing consultation to deaf people: a contextual analysis
}

Camila Crisse Justino de Araújo ${ }^{1}$, Alexsandro Silva Coura ${ }^{1}$, Inacia Sátiro Xavier de França ${ }^{1}$, Andressa Kaline Ferreira Araújoº Kaio Keomma Aires Silva Medeiros ${ }^{1}$

${ }^{1}$ Curso de Graduação em Enfermagem, Universidade Estadual da Paraíba (UEPB) - Campina Grande (PB), Brasil.

DOI: http://dx.doi.org/10.7322/abcshs.v40i1.702

\section{RESUMO}

Objetivou-se analisar criticamente os aspectos contextuais que influenciam a realização da consulta de Enfermagem às pessoas surdas. Estudo de revisão crítica-narrativa da literatura, desenvolvido de julho a agosto de 2013, nas bases de dados SciELO, LILACS e IBECS, assim como no Google Acadêmico e em portais eletrônicos do Ministério da Saúde, da Organização Mundial de Saúde e do Instituto Brasileiro de Geografia e Estatística, procedendo-se à análise do material coletado a partir do método de Análise Contextual. Diante do corpus de publicações selecionadas, depreenderam-se temas agrupados em camadas interativas de contexto, contemplando: particularidades da consulta de Enfermagem às pessoas surdas; obstáculos enfrentados em tal situação; compreensão sociocultural e psicológica da relação enfermeiro/paciente surdo/intermediador e legislação aplicada - bases para a prática da ética na consulta de Enfermagem às pessoas surdas. Concluiu-se que a consulta de Enfermagem ao paciente surdo não ocorre de forma satisfatória em virtude, principalmente, da inadequação de tais profissionais que, sem o devido preparo no âmbito acadêmico, negligenciam o cuidado a esta importante parcela da população.

Palavras-chave: enfermagem; cuidados de enfermagem; surdez.

\section{ABSTRACT}

This study aimed at critically analyzing the contextual factors that influence Nursing consultation to deaf people. It is a critical review of literature, developed from July to August 2013, on SciELO, LILACS and IBECS databases as well as on Google Scholar and electronic portals of the Ministry of Health, of the World Health Organization and of Instituto Brasileiro de Geografia e Estatística - IBGE. Then, we proceeded to the analysis of the material collected using the Contextual Analysis method. Given the corpus of selected publications, investigated subjects were grouped into interactive layers of context, comprising: peculiarities of Nursing consultation to deaf people; obstacles faced in Nursing consultation to deaf people; sociocultural and psychological understanding of the nurse/deaf patient/intermediator; and law applied - bases for ethics practice in Nursing consultations to deaf people. It was concluded that Nursing consultation to deaf patients does not occur satisfactorily due mainly to the inadequacy of nurses, without the correct preparation in the academic area, overlooking the care to this important segment of the population.

Keywords: nursing; nursing care; deafness. 


\section{INTRODUÇÃO}

A preocupação com os segmentos populacionais em vulnerabilidade no contexto brasileiro passou a ter maior atenção no final do século passado. Dentre esses grupos, as pessoas com deficiência auditiva encontram barreiras para realizar atividades de autocuidado e acesso à saúde e aos bens e serviços ${ }^{1}$.

No Brasil, cerca de 9,7 milhões de brasileiros apresentam essa condição. Deste total, dois milhões apresentam deficiência auditiva severa (1,7 milhões têm grande dificuldade para ouvir e 300.000 são surdos). O Estado da Paraíba, em especial, ocupa o segundo lugar no ranking dos estados com a maior proporção de indivíduos com deficiência, tendo mais de 48.000 pessoas com grandes dificuldades ou incapacidade de ouvir².

A surdez pode ser definida como a perda total ou parcial da acuidade auditiva ${ }^{3}$, unilateral ou bilateralmente de $41 \mathrm{~dB}$ ou mais. De uma forma mais ampla, a literatura dispõe de dois grandes paradigmas de compreensão sobre a deficiência, a saber: clínico-terapêutico e socioantropológico. Para o primeiro, a noção de deficiência é central e, dessa forma, foca-se na reabilitação e na cura, bem como no aprendizado da linguagem oral. Por outro lado, o segundo propõe que a surdez seja vista como uma diferença cultural, tal como à de outras minorias étnicas e linguísticas, entendendo que os surdos não são deficientes, e, portanto, não se aspira à cura, mas sim à valorização de seus direitos, cultura e comunidade ${ }^{4}$.

Desconsiderando-se a filosofia de abordagem dos surdos, se deficientes ou como uma minoria social, o que parece ser consenso é que esse tipo de condição é de difícil convívio, porquanto a audição é o sentido essencial para a aquisição e o uso da linguagem. Ao se comparar, sem a pretensão de estabelecer graus de importância ou prioridade, as pessoas com deficiências física e visual e o surdo, este é o que enfrenta maiores dificuldades de inclusão na sociedade ${ }^{3}$.

Essa constatação impacta a assistência em saúde, visto que, ante a limitação de se comunicar, é comum o surdo decidir por não ir a um serviço de saúde, inviabilizando a tradicional relação profissional e paciente. Nesse contexto, um marco legal importante é o decreto 5.626/05, que regulamenta a Lei 10.436, dispondo sobre a Língua Brasileira de Sinais (Libras), considerando-a como um meio de comunicação e expressão, bem como recomendando às instituições públicas de assistência à saúde garantir atendimento e tratamento adequado aos surdos 5 .

Não obstante, apesar dos esforços empreendidos no sentido de estabelecer parâmetros legais para o uso da Libras, sobretudo no âmbito da saúde, acredita-se que a comunicação com essa população permanece negligenciada neste setor, incluindo-se, portanto, a Enfermagem, na medida em que a assistência prestada pelo enfermeiro se dá, principalmente, por meio da comunicação estabelecida com o paciente no momento da consulta ${ }^{6,7}$.

A consulta de Enfermagem é uma atividade privativa dos enfermeiros, utilizada para identificar os problemas e as potencialidades dos usuários, a fim de decidir sobre um plano de cuidados que atenda às necessidades apresentadas pelo cliente, bem como para avaliar as intervenções desenvolvidas ${ }^{8}$. Assim sendo, cabe assinalar que não implementá-la, ou fazê-la de forma equivocada, dificulta e, não raro, impossibilita a identificação de problemas e possíveis soluções.

Ainda que ocorra a mediação da consulta de Enfermagem ao surdo por terceiros, como parentes, amigos ou até mesmo um intérprete profissional, entende-se que a interação não é otimizada, tendo em vista que, vencido o problema da comunicação, outro tão importante quanto é a dificuldade em resguardar a privacidade do paciente ${ }^{7}$.

Por esse motivo, a Libras é um recurso de comunicação que precisa ser conhecido e valorizado na prática das ações em saúde. Mesmo que isso não seja possível a curto prazo, é fundamental interpretar seus aspectos suprassegmentais que incluem gestos, expressões faciais e corporais para atenuar limitações expostas durante $\mathrm{o}$ atendimento ${ }^{7}$.

Além disso, acredita-se que abranger fenômenos contextuais que envolvem as pessoas surdas, tal como propõe este estudo, é imprescindível para a realização de uma assistência efetiva. Isso porque a interação do profissional de saúde com o contexto aumenta a exatidão e perfeição das interpretações, amplia o valor explicativo dos resultados, cria condições para a compreensão dos processos da vida humana e permite compartilhar seu significado e sua compreensão?.

Levando em conta que a assistência de Enfermagem ocorre, sobretudo, por meio da consulta e comunicação estabelecida com o cliente e que, por meio dela, pode-se compreendê-lo como um ser holístico, percebendo seu entendimento, suas sensações e seu modo de agir e reagirí ${ }^{6}$, indica-se a pertinência desta investigação, vislumbrando-a como um meio de reconhecer, enfrentar e superar as lacunas existentes na comunicação entre enfermeiros e surdos, a começar pela escassez de estudos que abordam a temática proposta.

Ademais, por meio desta investigação, espera-se oferecer mais conhecimento, contribuindo no tocante à formação dos profissionais e consequente melhoria na qualidade do atendimento ao referido público. Outrossim, espera-se subsidiar estratégias para melhorar o desempenho dos enfermeiros, estabelecendo de maneira eficaz práxis de Enfermagem no que diz respeito à consulta às pessoas surdas.

Objetivou-se analisar criticamente os aspectos contextuais que influenciam a realização da consulta de Enfermagem às pessoas surdas.

\section{MÉTODOS}

Estudo de revisão crítica-narrativa da literatura científica, com abordagem qualitativa, realizado em julho e agosto de 2013, cujos dados foram coletados nas bases de dados SciELO, LILACS e IBECS, assim como no Google Acadêmico e em websites oficiais do Ministério da Saúde do Brasil, do Instituto Brasileiro 
de Geografia e Estatística (IBGE) e da Organização Mundial de Saúde (OMS).

A partir dos descritores em ciências da saúde "Consulta de Enfermagem", "Deficiência” e "Pessoas Surdas", coletaram-se múltiplos e variados tipos de publicações, dentre elas artigos científicos, teses, dissertações, monografias e relatórios, uma vez que possuíssem, em seus títulos, no mínimo um dos descritores retromencionados, bem como estivessem disponíveis, de forma gratuita e integralmente, na Internet. Foram excluídas as publicações que, embora atendessem aos referidos critérios, não possuíam potencial para o alcance do objetivo proposto, verificado por meio da leitura de seus resumos.

Uma vez coletados, foram armazenados e, posteriormente, efetuaram-se sucessivas leituras flutuantes dos textos, de modo a possibilitar um contato mais aprofundado do pesquisador com as informações constantes neles, permitindo-se fluir interpretações gerais. Cabe assinalar, contudo, que inicialmente não houve a pretensão de sistematizar tais interpretações, mas sim compreender, sob uma perspectiva global, o teor das publicações coletadas.

Após serem coletadas as informações centrais das publicações, conforme descrito, seguiu-se à análise propriamente dita, a qual foi realizada sob a ótica da Análise Contextual, cuja essência e especificidade, conforme apontam seus teóricos, se traduz à compreensão do fenômeno estudado. Neste caso, a consulta de Enfermagem à pessoa surda, a partir de quatro camadas interativas de contexto 9 .

Nessa perspectiva, o desenvolvimento deste estudo esteve pautado na compreensão de informações à luz das seguintes camadas interativas de contexto e suas principais características, respectivamente: imediata (focaliza o presente, englobando os dados contextuais mais aparentes da imediação em que o fenômeno ocorre); específica (envolve o passado imediato e os fatores relevantes da condição no momento em que está ocorrendo); geral (considera a compreensão de vida dos sujeitos envolvidos no fenômeno, gerada por meio das interações passadas e atuais com aquela situação) e, por fim, metacontexto (incorpora passado e presente e molda o futuro, sendo uma fonte de conhecimento socialmente construído e que opera continuamente, resultando em uma perspectiva social compartilhada) ${ }^{9}$.

Os dados coletados foram sumarizados e delimitados conforme a perspectiva conceitual de cada camada de contexto atingida. Cabe assinalar, contudo, que elas são interativas e não estáticas e/ou isoladas, estando apresentadas em subtemas para facilitar a visualização e, consequentemente, a compreensão do todo, com o intuito de se atingir o meio gestalt do fenômeno. Ao propor este modelo de análise, o pesquisador compreende com mais facilidade o seu objeto de estudo, porquanto consegue propor uma sequência lógica de análise, a partir da qual suas interpretações vão sendo postas de forma não aleatória, mas sim guardando certa sistematização.

\section{RESULTADOS E DISCUSSÃO}

Os subtemas foram configurados da seguinte maneira: as particularidades da consulta de Enfermagem às pessoas surdas - contexto imediato; os obstáculos enfrentados em tal situação - específico; a compreensão sociocultural e psicológica da relação enfermeiro/paciente surdo/intermediador - geral; a legislação aplicada: bases para a prática da ética na consulta de enfermagem às pessoas surdas - metacontexto. As camadas contextuais, representadas por seus respectivos subtemas, estão dispostas na Figura 1.

METACONTEXTO - Legislação aplicada: bases para a prática da ética na consulta de Enfermagem às pessoas surdas

- Política Nacional para a Integração da Pessoa Portadora de Deficiência e Política Nacional de Saúde da Pessoa com Deficiência

- Política Nacional de Atenção Básica

- Princípios do Sistema Único de Saúde

- Paradigma biomédico versus Formação profissional

CONTEXTO GERAL - Compreensão sociocultural e psicológica da relação enfermeiro/paciente surdo/intermediador

- Sentimentos negativos do surdo: insatisfação, descontentamento e descrédito;

- Sensação de ser tratado com preconceito;

- Fatores subjetivos;

- Compreensão sociocultural e psicológica da relação enfermeiro/paciente surdo/intermediador;

- Valores e sentimentos do próprio profissional acerca do atendimento, podendo esse se sentir frustrado, impotente ou impaciente.

CONTEXTO ESPECÍFICO - Obstáculos enfrentados na consulta de Enfermagem às pessoas surdas

- Déficit dos profissionais de saúde no atendimento;

- Dificuldade na comunicação entre enfermeiros e indivíduos surdos;

- Falta de competência técnica por parte do profissional de saúde;

- Tempo insuficiente para prestar uma assistência de qualidade.

CONTEXTO IMEDIATO - Particularidades da consulta de Enfermagem às pessoas surdas

- Processo comunicativo com pacientes surdos é prejudicado;

- Profissional de saúde precisa estar devidamente capacitado para prestar assistência digna e efetiva;

- Necessidade da utilização de intérpretes para facilitar o atendimento:

- Transmissão das informações pode ser afetada pela falta de preparo dos profissionais envolvidos na assistência.

Figura 1: Representação esquemática das camadas contextuais da consulta de Enfermagem às pessoas surdas 


\section{As particularidades da consulta de Enfermagem às pessoas surdas}

Normalmente, a relação dos profissionais de saúde com pacientes que têm audição em níveis normais é estabelecida pela linguagem oral. Contudo, dependendo do grau de surdez, os pacientes surdos não têm como utilizarem-se desse mecanismo ${ }^{7}$. Portanto, não se pode prescindir da importância do processo comunicativo na relação profissional-paciente.

Destarte, a comunicação efetiva é condição imprescindível para que o profissional de saúde, especialmente o enfermeiro, possa auxiliar o paciente a atender suas demandas em saúde. Para tanto, é essencial o uso adequado das técnicas de comunicação interpessoal $^{10}$. No momento em que o receptor da mensagem transmitida, nesse caso o enfermeiro, torna-se vulnerável e não pode efetivamente compreender o que é dito pelo emissor, sendo este o paciente surdo, o processo de comunicação é comprometido e, como consequência, a resposta pode ser inadequada ou não corresponder ao que era esperado ${ }^{11}$. Desse modo, torna-se premente a capacitação dos profissionais de saúde para prestarem assistência de qualidade, na perspectiva de minimizar as incapacidades e facilitar a inclusão do indivíduo na sociedade.

Nessa perspectiva, considerando-se que, na maioria das vezes, os surdos fazem uso de leitura labial, o profissional deve falar de forma que o cliente possa ler seus lábios, frente à frente, possibilitando a visualização dos movimentos da boca, evitando, inclusive, cobri-la com as mãos, possibilitando a total compreensão da informação transmitida, falando lentamente e com clareza. Assim, um ambiente com iluminação adequada favorece uma melhor compreensão ${ }^{12}$.

Ademais, os surdos nem sempre conseguem ler o que foi escrito pelo profissional de saúde porque existem palavras difíceis, termos técnicos, pela deficiência do surdo no que concerne ao português ou simplesmente porque a letra do profissional é ilegível. A utilização da escrita pode ser proveitosa durante a consulta de Enfermagem para a comunicação com esses pacientes. No entanto, é comum a população surda ter menos instrução do que o restante. Sendo assim, é importante que, para explicações complexas, sejam dispensados maior atenção e cuidados quando da utilização de termos técnicos para tais explicações ${ }^{13}$.

O emprego de profissionais intérpretes se faz necessário muitas vezes. Todavia, existem algumas críticas e limitações para a atuação desses. A presença deles durante o atendimento pode provocar ou aumentar o constrangimento, colocar em risco o direito de sigilo e privacidade, bem como prejudicar a qualidade das informações repassadas. Ademais, o envolvimento de intérpretes limita o estabelecimento do vínculo profissional-paciente e, nessas condições, entende-se que há dificuldade de se estabelecer uma assistência e, particularmente, uma consulta adequada ${ }^{6}$.

Outrossim, os embaraços que o enfermeiro apresenta ao se deparar com o paciente surdo interferem negativamente em seu cuidar. A dificuldade desta clientela em receber a abordagem e o tratamento adequados se justifica, principalmente, pela falta de preparo, paciência, compreensão e inclinação de tal profissional para com o seu cliente surdo ${ }^{14}$.

Assim, entende-se que o contexto imediato da consulta de Enfermagem às pessoas surdas está permeado por peculiaridades e especificidades, fato que gera a necessidade dos enfermeiros implementarem ações diferenciadas em comparação com a assistência aos demais usuários do sistema de saúde.

\section{Obstáculos enfrentados na consulta de Enfermagem às pessoas surdas}

Há múltiplos e variados obstáculos inerentes à consulta ao paciente surdo, destacando-se: as dificuldades linguísticas; a falta de confiança no mundo dos que ouvem; o fato de se comparar os surdos com deficientes mentais, considerando-os como pessoas com baixo nível intelectual; a falta de acesso às informações preventivas; o pouco conhecimento acerca da assistência em saúde e o menor índice de frequência aos serviços de atenção básica ${ }^{7}$.

Diante do que foi colocado, depreende-se que a acessibilidade aos serviços de saúde pode ser afetada negativamente pela presença da surdez. Intervenções específicas e dirigidas para o grupo da população com esta condição, bem como a avaliação das políticas públicas atuais, colocando-se em prática tudo o que é garantido pelas leis vigentes, representariam uma alternativa de minimizar as dificuldades encontradas, bem como promover a saúde integral na atenção básica ${ }^{11}$.

Desta forma, a comunicação não verbal é fundamental no atendimento aos sujeitos surdos e permite a excelência do cuidar em saúde. Isso porque o profissional que a reconhece apropriadamente potencializa sua interação com o paciente, algo desejado quando se pretende estabelecer uma relação de confiança. Entretanto, em geral, os profissionais utilizam sinais e gestos que acreditam ser adequados para transmitir ao surdo o que estão querendo expressar ou solicitam a ajuda do acompanhante para fazer a intermediação. Portanto, isso se configura como outro obstáculo para a manutenção da assistência, nesse caso, do enfermeiro, posto que as caracterizações dos comportamentos não verbais aplicados às populações de ouvintes não são completamente atribuíveis aos grupos de surdos ${ }^{13}$.

Entende-se que, no momento em que o surdo procura atendimento de saúde e está com sinais clínicos de doença, é mais fácil para o enfermeiro, até certo ponto, detectar o que está ocorrendo. Todavia, em doenças sem causa aparente, em que é necessário coletar, de modo ampliado, a história do paciente, a situação torna-se mais complicada, deixando evidente a carência dos profissionais no que diz respeito à preparação para o atendimento a esse público ${ }^{13}$.

Reitera-se que o enfermeiro tem de adquirir competência no uso de técnicas de comunicação não verbal, no intuito de desenvolver uma postura que permita a aquisição de conhecimentos das questões inerentes a um cuidado humanizado a todos os clientes, com o objetivo de lhes oferecer apoio, conforto, informação e despertar seus sentimentos de confiança e autoestima ${ }^{15}$. 
Apesar de a família representar um auxílio, na maioria das vezes também pode ser um problema, como quando não permite que o surdo participe como agente ativo de seu tratamento e explica ao profissional os problemas de saúde que o mesmo apresenta, sendo, por conseguinte, a pessoa que recebe as orientações. Assim, os surdos podem não ter uma oportunidade de exporem suas dúvidas e inquietações ${ }^{13}$.

Por fim, acrescenta-se que outro importante obstáculo para a consulta de Enfermagem às pessoas surdas se refere à falta de condições de trabalho adequadas, a exemplo do tempo insuficiente para os enfermeiros realizarem as atividades protocoladas como prioridades pelo Ministério da Saúde.

Portanto, configurando o contexto específico, percebe-se que a assistência que o surdo recebe por parte da equipe de Enfermagem não se assemelha ao que por lei lhe é de direito, sendo alguns desses: direito de participar na tomada de decisões sobre sua saúde; direito à informação atualizada, relevante e compreensível sobre seu diagnóstico, tratamento e prognóstico e de conhecer a identidade dos médicos, enfermeiros e demais envolvidos em seus cuidados; direito de ter intérprete quando seu idioma não é entendido; enfrentando tal assistência, ainda, muitos obstáculos para a sua efetivação ${ }^{14}$.

\section{Compreensão sociocultural e psicológica da relação enfermeiro/paciente surdo/intermediador}

A surdez, antigamente, era um fator discriminador. Tais indivíduos eram considerados dignos de pena e vítimas da incompreensão da sociedade e até mesmo da própria família. Contudo, essa visão vem se modificando e é atualmente discutida por profissionais das mais variadas áreas de conhecimento ${ }^{7}$.

A experiência dos profissionais ao cuidar desses clientes tem relação com a sua prática profissional ao longo dos anos. Discute-se sobre a deficiência como uma doença, porém não se buscam formas de o enfermeiro cuidar, se relacionar e se comunicar com o surdo $^{15}$. A capacitação dos profissionais da saúde para atender pacientes surdos é uma necessidade urgente que emerge da carência deles em entender a realidade vivenciada por esses pacientes. O problema de comunicação do indivíduo surdo não é orgânico, mas social e cultural. Estudos mostram que, para trabalhar com grupos dessa natureza, é essencial compreender sua cultura, linguagem e suas percepções ${ }^{16}$.

Pesquisas reportam que o processo de comunicação entre o profissional e o surdo gera medo, especialmente quando esse está desacompanhado para o atendimento em saúde. Outro receio é quanto ao preenchimento de documentos, haja vista a necessidade da realização dos protocolos de atendimento, uma vez que este sujeito nem sempre entende o que ali está descrito ${ }^{13}$.

O profissional moderno deve ser eficiente, prestativo, se preocupar com o relacionamento enfermeiro-usuário, de maneira holística, com o intuito de proporcionar estabilidade emocional ao paciente, para que desta forma ocorra, efetivamente, a promoção, a proteção e a recuperação da saúde ${ }^{14}$.
O surdo, na maioria das vezes, precisa de pessoas que traduzam suas emissões para os profissionais e vice-versa, emergindo, assim, a necessidade de intermediação. A figura do intermediador configura-se na família, nos amigos e/ou no intérprete profissional ${ }^{13}$. Quando a presença do intérprete é inviável, é gerado um bloqueio psicológico no indivíduo surdo, interferindo diretamente na assistência, o que aumenta o sofrimento causado em sua consulta ${ }^{16}$.

Vale ressaltar que o enfermeiro também é atingido psicologicamente durante a consulta ao paciente surdo. Ao tentar interagir com essas pessoas, os profissionais de Enfermagem e da área da saúde em geral podem apresentar sentimentos de frustração, impotência e impaciência, por não conseguirem desenvolver uma assistência adequada ${ }^{15}$.

Além dos percalços enfrentados durante a consulta de Enfermagem aos surdos, os usuários dos serviços públicos de saúde acreditam que a assistência gratuita é de baixa qualidade, que as instituições são desprovidas de equipamentos básicos e que seus problemas, consequentemente, não serão solucionados. Essa situação provoca sentimentos de insatisfação, descontentamento e descrédito nos usuários que, não obstante, utilizam os serviços ${ }^{17}$.

$\mathrm{O}$ deficiente auditivo tem suas limitações, mas não se deve tratá-lo, preconceituosamente, como um ser humano diferente. Suas limitações não podem impedir sua comunicação e seu relacionamento. Portanto, os profissionais precisam ser competentes no desempenho de seus papéis como cuidador, melhorando sua relação com o paciente surdo ${ }^{3}$.

Nesse sentido, acredita-se na existência de fatores subjetivos que podem interferir na realização da consulta de Enfermagem às pessoas surdas, tendo em vista a associação de eventos, comportamentos e acontecimentos a significados elaborados pelo indivíduo pelo tempo. Logo, observa-se a interligação dessa situação com o contexto mais geral do fenômeno estudado.

\section{Legislação aplicada: bases para a prática da ética na consulta de Enfermagem às pessoas surdas}

Conviver no universo das pessoas surdas envolve uma mudança de paradigmas. Para eles, essas transformações sucedem quando são aceitos e respeitados pela sociedade em suas diferenças, em sua condição ${ }^{16}$.

Os surdos se sentem discriminados não apenas por sua condição física, mas por não receberem atendimento adequado, como garantem as leis vigentes no Brasil. Sentem-se discriminados por esperarem muito tempo na fila para serem atendidos, já que não escutam seus nomes serem chamados, e o profissional, mesmo sabendo que o cliente não pode ouvir, não se atenta a chamá-lo de forma apropriada ${ }^{13}$.

O Brasil, no intuito de atender às demandas das pessoas com deficiência, instituiu a Política Nacional para a Integração da Pessoa Portadora de Deficiência e a Política Nacional de Saúde da Pessoa com Deficiência, dispondo, dentre outras diretrizes: assegurar os direitos básicos, inclusive à educação, à saúde e ao 
trabalho; promover a qualidade de vida; garantir o funcionamento dos serviços de atenção à pessoa com deficiência e fomentar a capacitação de recursos humanos para assistir essas pessoas. Essas peças jurídicas estão respaldadas pelos princípios do Sistema Único de Saúde (SUS), o qual preconiza a equidade, integralidade e universalidade ${ }^{18-20}$.

A Atenção Primária, especificamente, engloba um conjunto de ações que visam à promoção da saúde, à prevenção, ao tratamento de doenças e à redução de danos ou sofrimentos que venham a comprometer a convivência de modo saudável. Considera-se o sujeito em sua singularidade, complexidade, integralidade e na inserção sociocultural. Entre os entraves verificados na assistência às pessoas surdas, em especial na Atenção Básica, ainda tem destaque o modelo biomédico, caracterizado pela tecnicidade da Medicina, enfocando valores fisiológicos e analíticos em detrimento de fatores psicossociais que possam afetar o paciente. Nessa perspectiva, as ações de Enfermagem também apresentam interfaces mecanicistas e reducionistas ${ }^{21}$.

O surdo é quem enfrenta maior dificuldade de inclusão na sociedade, em comparação às pessoas com deficiências física e visual, uma vez que a audição é o sentido essencial para o desenvolvimento e uso da linguagem. A surdez se configura como um distúrbio neurológico sensorial que afeta a possibilidade de comunicação oral e de aprendizagem deste tipo de clientela ${ }^{22}$.

O capítulo VII do Decreto de Lei 5.626 trata da "garantia do direito à saúde das pessoas surdas ou com deficiência auditiva" e determina que, a partir de 2006, o atendimento às pessoas surdas ou com deficiência auditiva na rede de serviços do SUS, bem como nas empresas que detêm concessão ou permissão dos serviços públicos de assistência à saúde, seja realizado por profissionais capacitados para o uso da Libras ou para a sua tradução e interpretação $0^{5}$.

A luta da comunidade surda brasileira que utiliza a Libras resultou na aprovação da Lei Federal 10.436/02, encarregando as instituições públicas de oportunizarem programas que visem à preparação dos profissionais da área de saúde para o atendimento e tratamento de pacientes surdos ${ }^{7}$.

Está em atividade, atualmente no Brasil, o curso de graduação em Letras - Libras, ofertado em 18 polos, abrangendo as cinco regiões brasileiras. Em relação aos tradutores-intérpretes, desde 2007, o Ministério da Educação realiza anualmente o Exame Nacional para Certificação de Proficiência em Libras e para Certificação de Proficiência em Tradução e Interpretação de Libras (Prolibras), que certificaram 6.100 profissionais. O exame está previsto para continuar sendo realizado até $2016^{20}$.

Aprender a Libras vai além da sala de aula, haja vista tratar-se de uma língua, o que exige um contexto e contato com a cultura em questão. Aponta-se a relevância dos cursos na área de saúde atentarem para uma visão mais ampla e acolhedora na implantação do componente Libras em seus currículos ${ }^{6}$.
Considerando-se a complexidade da relação do profissional de saúde com pacientes surdos, é fundamental conhecer os aparatos legais que a regem. Compreender a identidade da pessoa surda e os fatores culturais que a caracterizam são diferenciais imprescindíveis na qualidade dos serviços prestados a essa população ${ }^{7}$.

Assim, acredita-se na necessidade de mudanças na formação dos profissionais, objetivando-se alcançar um perfil mais humanizado, reflexivo e com ênfase na universalidade e integralidade das ações. Nessa perspectiva, entende-se ter contemplado o metacontexto do fenômeno estudado, abrangendo outros níveis contextuais.

\section{CONCLUSÃO}

Este estudo possibilitou uma maior compreensão acerca da consulta de Enfermagem às pessoas surdas, incentivando uma reflexão sobre os desafios enfrentados, quer pelo paciente surdo, quer pelo profissional de saúde.

Apesar de o conhecimento derivado das pesquisas científicas ser relevante para o planejamento de políticas públicas e para a reorganização das atividades assistenciais, percebeu-se que há uma escassez de estudos acerca da assistência de enfermagem às pessoas surdas, configurando-se como uma grande perda.

Sendo a comunicação um instrumento indispensável à prática em saúde, particularmente em Enfermagem, constatou-se que o emprego deste pelos enfermeiros não é eficiente, quando se trata de pessoas surdas. É indubitável, portanto, que a consulta de Enfermagem a esse público não poderá alcançar a excelência enquanto essa lacuna continuar sendo verificada.

Desse modo, há de se considerar, conforme aponta o presente estudo, que existem inúmeros fatores concorrendo para a dificuldade na consulta do enfermeiro ao surdo, tais como: circunstâncias imediatas, como a falta de preparo dos profissionais de saúde para lidar com os surdos e a dificuldade de comunicação entre eles; condições específicas que geram dificuldades no atendimento, como condições desfavoráveis de trabalho, limitações do surdo; situações mais gerais, por exemplo os sentimentos negativos que permeiam a consulta, os valores, as compreensões por parte do surdo e do enfermeiro e, finalmente, condições metacontextuais representadas pelas políticas públicas.

Finalmente, percebe-se que o incentivo à implementação das políticas públicas é um fator imprescindível no fortalecimento da prática profissional, em detrimento do modelo biomédico, haja vista a existência de barreiras que impossibilitam a prestação de uma assistência de qualidade. Na consulta de Enfermagem às pessoas surdas, especificamente, essa implementação possibilitará o exercício pleno dos direitos e da cidadania do indivíduo surdo, levando-o a assumir a corresponsabilidade no concernente à sua própria vida e saúde. 


\section{REFERÊNCIAS}

1. Souza MT, Porrozzi R. Ensino de libras para os profissionais de saúde: uma necessidade premente. Rev Práxis. 2009:1(2):43-6.

2. Instituto Brasileiro de Geografia e Estatística (IBGE). Censo Demográfico: características da população e dos domicílios. Rio de Janeiro: Gráfica digital; 2011. 270p.

3. Pagliuca LM, Fiuza NL, Reboucas CB. Aspectos da comunicação da enfermeira com o deficiente auditivo. Rev Esc Enferm USP. 2007:41(3):411-8.

http://dx.doi.org/10.1590/S0080-62342007000300010

4. Bisol C, Sperb TM. Discursos sobre a surdez: deficiência, diferença, singularidade e construção de sentido. Psicol Teor Pesqui. 2010;26(1):7-13

5. Brasil [Internet]. Decreto $n^{\circ} 5.626$, de 22 de dezembro de 2005. Regulamenta a Lei no 10.436, de 24 de abril de 2002, que dispõe sobre a Língua Brasileira de Sinais - Libras. 2005. Disponível em: http://www.planalto.gov.br/ccivil_03/_ato2004-2006/2005/decreto/ d5626.htm. Acesso em: 04 out. 2013

6. Oliveira YC, Costa GM, Coura AS, Cartaxo RO, França IS. A língua brasileira de sinais na formação dos profissionais de enfermagem, fisioterapia e odontologia no estado da Paraíba, Brasil. Interface: Com Saúde Educ. 2012;16(43):995-1008. http://www.dx.doi.org/10.1590/S1414-32832012005000047

7. Chaveiro N, Barbosa MA, Porto CC. Revisão de literatura sobre o atendimento ao paciente surdo pelos profissionais da saúde. Rev Esc Enferm USP. 2008;42(3):578-83. http://dx.doi.org/10.1590/S0080-62342008000300023

8. Conselho Federal de Enfermagem (COFEN) [Internet]. Resolução $n^{\circ}$ 317/2007. Revoga a Resolução COFEN-271/2002 que dispõe sobre as ações do Enfermeiro na consulta, prescrição de medicamentos e requisição de exames. Disponível em: http://novo.portalcofen.gov. br/resoluo-cofen-3172007_4351.html. Acesso em: 04 out. 2013.

9. Hinds PS, Chaves DE, Cypress SM. Context as a source of meaning and understanding. Qual Health Res. 1992;2(1):61-74. http://10.1177/104973239200200105

10. Braga EM, Silva MJP. Comunicação competente - visão de enfermeiros especialistas em comunicação. Acta Paul Enferm. 2007:20(4):410-4.

http://dx.doi.org/10.1590/S0103-21002007000400004

11. Castro SS, Paiva KM, Cesar CL. Communication difficulties between individuals with hearing disability and health professionals: a public health matter. Rev Soc Bras Fonoaudiol. 2012;17(2):128-34. http://dx.doi.org/10.1590/S1516-80342012000200005
12. Oliveira HR, Lopes KS, Pinto NM. Percepção da equipe de enfermagem acerca da assistência prestada ao deficiente auditivo. Rev Enferm Integrada. 2009;2(1):165-75.

13. Cardoso AH, Rodrigues KG, Bachion MM. Percepção da pessoa com surdez severa e/ou profunda acerca do processo de comunicação durante seu atendimento de saúde. Rev Latino-Am Enferm. 2006;14(4):553-60. http://dx.doi.org/10.1590/S0104-11692006000400013

14. Corrêa CS, Pereira LA, Barreto LS. Celestino PP, André KM. O despertar do enfermeiro em relação ao paciente portador de deficiência auditiva. Rev Pesqui Cuid Fundam. 2010;2(2):758-69.

15. Gomes V, Soares MC, Muniz RM, Silva JRS. Vivência do enfermeiro ao cuidar surdos e/ou portadores de deficiência auditiva. Enferm Glob. 2009;17.

http://dx.doi.org/10.4321/S1695-61412009000300007

16. Chaveiro N, Barbosa MA. Assistência ao surdo na área de saúde como fator de inclusão social. Rev Esc Enferm USP. 2005;39(4):417-22.

17. Costa RK, Enders BC, Menezes RM. Trabalho em equipe de saúde: uma análise contextual. Ciência Cuid Saúde. 2008;7(4):530-6.

18. Brasil. Decreto $n^{\circ} 3.298$, de 20 de dezembro de 1999. Regulamenta a Lei no 7.853, de 24 de outubro de 1989, dispõe sobre a Política Nacional para a Integração da Pessoa Portadora de Deficiência, consolida as normas de proteção, e dá outras providências. Brasília; 1999

19. Brasil. Ministério da Saúde. Secretaria de Atenção à Saúde. Política Nacional de Saúde da Pessoa Portadora de Deficiência. Brasília; 2008.

20. Brasil. Diário Oficial da União. Portaria 2.488 de 21 de outubro de 2011. Aprova a Política Nacional de Atenção Básica, estabelecendo a revisão de diretrizes e normas para a organização da Atenção Básica, para a Estratégia Saúde da Família (ESF) e o Programa de Agentes Comunitários de Saúde (PACS). Brasília: DOU; 2011.

21. Raimundo RJS, Santos TA. A importância do aprendizado da comunicação em libras no atendimento ao deficiente auditivo em serviço de saúde. Renefra. 2012;3(3):184-91.

22. Brasil. Decreto $\mathrm{n}^{\circ}$ 7.612, de 17 de Novembro de 2011. Institui o Plano Nacional dos Direitos da Pessoa com Deficiência. Plano Viver sem Limite. Brasília; 2011. 